\title{
ON THE OSCILLATION OF A THIRD ORDER NONLINEAR DIFFERENTIAL EQUATIONS WITH NEUTRAL TYPE
}

\author{
V. Ganesan \\ Department of Mathematics, Aringar Anna Government Arts College, \\ Namakkal, Tamilnadu, India \\ ganesan_vgp@rediffmail.com

\section{Sathish Kumar} \\ Department of Mathematics, Paavai Engineering College (Autonomous), \\ Namakkal, Tamilnadu, India \\ msksjv@gmail.com
}

\begin{abstract}
In this article, we investigate the oscillation behavior of the solutions of the third-order nonlinear differential equation with neural type of the form$$
\left(a_{1}(t)\left(a_{2}(t) Z^{\prime}(t)\right)^{\prime}\right)^{\prime}+q(t) f(x(\sigma(t)))=0, \quad t \geq t_{0}>0,
$$

where $Z(t):=x(t)+p(t) x^{\alpha}(\tau(t))$. Some new oscillation results are presented that extend those results given in the literature.

AMS Subject Classification: 34C10, 34K11.

Key words: Oscillation, Non-linear, Neutral differential equation, Third order.
\end{abstract}

\section{Introduction}

Consider the third order non-linear neutral delay differential equation

$$
\left(a_{1}(t)\left(a_{2}(t) Z^{\prime}(t)\right)^{\prime}\right)^{\prime}+q(t) f(x(\sigma(t)))=0, \quad t \geq t_{0}>0,
$$

where $Z(t):=x(t)+p(t) x^{\alpha}(\tau(t))$ and $0<\alpha \leq 1$ is a ratio of odd positive integers. Throughout this paper, without further mention, let

$\left(A_{1}\right) a_{i}(t) \in C\left(\left[t_{0},+\infty\right)\right), a_{i}(t)>0$ for $i=1,2$ and $p(t), q(t) \in C\left(\left[t_{0},+\infty\right)\right), q(t)>0$;

$\left(A_{2}\right) \tau(t) \in C\left(\left[t_{0},+\infty\right)\right), \tau(t) \leq t, \sigma(t) \in C\left(\left[t_{0},+\infty\right)\right), \sigma(t) \leq t$

$\left(A_{3}\right) \quad f$ is nondecreasing and $u f(u) \geq k>0$ for $u \neq 0$ and $\lim _{t \rightarrow+\infty} \tau(t)=\lim _{t \rightarrow+\infty} \sigma(t)=\infty$.

By a solution of equation $(E)$ we mean a nontrivial real valued function $x(t) \in C\left(\left[T_{x}, \infty\right)\right), T_{x} \geq$ $t_{0}$, which has the property $Z^{\prime}(t) \in C^{1}\left(\left[T_{x}, \infty\right)\right), a_{2}(t) Z^{\prime}(t) \in C^{1}\left(\left[T_{x}, \infty\right)\right), a_{1}(t)\left(a_{2}(t) Z^{\prime}(t)\right)^{\prime} \in$ $C^{1}\left(\left[T_{x}, \infty\right)\right)$ and satisfies $(E)$ on $\left[T_{x}, \infty\right)$. We consider only those solutions $x(t)$ of $(E)$ which satisfy $\sup \{|x(t)|: t \geq T\}>0$ for all $T \geq T_{x}$. A solution of $(E)$ is called oscillatory if it has arbitrarily large zeros on $\left[T_{x}, \infty\right)$ and otherwise, it is said to be non-oscillatory. Equation $(E)$ is called almost oscillatory if all its solutions are oscillatory or convergent to zero asymptotically.

In the last years, a great deal of interest in oscillatory properties of neutral functional differential equations has been shown, we refer the reader to [1-8] and the references cited therein. A number 
of authors including B. Baculíková and J. Džurina [10], T. Candan and Dahiya [11, 12], Graef et al. [7], and E. Thandapani and Li [8] have studied the oscillatory behavior of solutions of third order neutral differential equations in the form of equation $(E)$ when $\alpha=1$.

Recently, Lin and Tang [13] explored the oscillation of first-order neutral differential equation with a super-linear neutral term

$$
\left[x(t)-p\left(x^{\alpha}(t-\tau)\right]^{\prime}+q(t) \prod_{j=1}^{m}\left|x\left(t-\sigma_{j}\right)\right|^{\beta_{j}} \operatorname{sgn}\left[x\left(t-\sigma_{j}\right)\right]=0,\right.
$$

where $\alpha>1$. Ravi P. Agarwal et al. [14] concerned with oscillation of a certain class of second-order differential equations with a sub-linear neutral term

$$
\left(a(t)\left[x(t)+p(t) x^{\alpha}(\tau(t))\right]^{\prime}\right)^{\prime}+q(t) x(\sigma(t))=0, \quad t \geq t_{0}>0,
$$

where $0<\alpha \leq 1$ is a ratio of odd positive integers and E. Thandapani et al. [9] established sufficient conditions for the oscillation of all solutions of a nonlinear differential equation

$$
\left(a(t)\left[x(t)+p(t) x^{\alpha}(\tau(t))\right]^{\prime}\right)^{\prime}+q(t) x^{\beta}(\sigma(t))=0, \quad t \geq t_{0}>0,
$$

where $\alpha$ and $\beta$ are ratio of odd positive integers. The above observation shows that this paper extend the results in third order.

This article presents the further investigation of the oscillations of $(E)$. The following two cases:

$$
\begin{aligned}
& \int_{t_{0}}^{\infty} \frac{1}{a_{1}(t)} d t=\infty, \quad \int_{t_{0}}^{\infty} \frac{1}{a_{2}(t)} d t=\infty, \\
& \int_{t_{0}}^{\infty} \frac{1}{a_{1}(t)} d t<\infty, \quad \int_{t_{0}}^{\infty} \frac{1}{a_{2}(t)} d t=\infty,
\end{aligned}
$$

are studied.

The paper is organized as follows. In Section 2, we present sufficient conditions for the oscillation of all solutions of $(E)$ and in Section 3, we provide some examples to illustrate the main results.

In the following, all functional inequalities considered in this paper are assumed to hold eventually, that is, they are satisfied for all $t$ large enough. Without loss of generality, we can deal only with the positive solutions of $(E)$.

\section{Main result}

In this section, we state and prove our main results for the equation $(E)$. For convenience, we use the notations

$$
p_{*}(t)=\left(1-\frac{p(\sigma(t))}{M^{1-\alpha}}\right), \quad \Theta(t)=\frac{\int_{t_{2}}^{\sigma(t)}\left(\frac{1}{a_{2}(s)} \int_{t_{1}}^{s} \frac{d u}{a_{1}(u)}\right) d s}{\int_{t_{1}}^{t} \frac{d u}{a_{1}(u)}}
$$

Theorem 1. Let $0 \leq p(t) \leq p_{1} \leq 1$. If (1.1) holds and it there exists a positive function $\phi \in C^{1}\left(\left[t_{0}, \infty\right), \mathbb{R}\right)$, such that for all sufficiently large $t_{3}>t_{2}>t_{1} \geq t_{0}$ we have

$$
\limsup _{t \rightarrow \infty} \int_{t_{3}}^{t}\left(\phi(s) k q(s) p_{*}(s) \Theta(s)-\frac{a_{1}(s)\left(\phi^{\prime}(s)\right)^{2}}{4 \phi(s)}\right) d s=\infty
$$


and

$$
\int_{t_{0}}^{\infty} \frac{1}{a_{2}(v)} \int_{v}^{\infty} \frac{1}{a_{1}(u)}\left[\int_{u}^{\infty} q(s) d s\right] d u d v=\infty
$$

holds for all constants $M>0$, then $(E)$ is almost oscillatory.

P r o o f. Suppose that $x(t)$ is a positive solution of $(E)$. By condition (1.1), there exist two possible cases:

(1) $Z(t)>0, Z^{\prime}(t)>0,\left(a_{2}(t) Z^{\prime}(t)\right)^{\prime}>0,\left(a_{1}(t)\left(a_{2}(t) Z^{\prime}(t)\right)^{\prime}\right)^{\prime}<0$,

(2) $Z(t)>0, Z^{\prime}(t)<0,\left(a_{2}(t) Z^{\prime}(t)\right)^{\prime}>0,\left(a_{1}(t)\left(a_{2}(t) Z^{\prime}(t)\right)^{\prime}\right)^{\prime}<0$, for $t \geq t_{1}, t_{1}$ is large enough.

Assume $Z(t)$ satisfying property (1), then

$$
\left(a_{1}(t)\left(a_{2}(t) Z^{\prime}(t)\right)^{\prime}\right)^{\prime}=-q(t) f(x(\sigma(t))) \leq-k q(t) x(\sigma(t))<0 .
$$

If there exists $t \geq t_{1}$ such that $Z(t)>0, Z(\sigma(t))>0, Z^{\prime}(t)>0$, then $Z(t)$ is monotonically increasing, there exists a constant $M>0$ such that $Z(t) \geq M$ and by the definition of $Z$ we have

$$
x(t)=Z(t)-p(t) x^{\alpha}(\sigma(t)) \geq Z(t)-p(t) Z^{\alpha}(\sigma(t)) \geq\left(1-\frac{p(\sigma(t))}{M^{1-\alpha}}\right) Z(t)=p_{*}(t) Z(t),
$$

where $p_{*}(t)$ is defined in (2.1). Let

$$
\omega(t)=\phi(t) \frac{a_{1}(t)\left(a_{2}(t) Z^{\prime}(t)\right)^{\prime}}{a_{2}(t) Z^{\prime}(t)}
$$

$\omega(t)>0$ for $t \geq t_{1}$. Differentiating (2.5), we obtain

$$
\begin{aligned}
\omega^{\prime}(t)= & \phi^{\prime}(t) \frac{a_{1}(t)\left(a_{2}(t) Z^{\prime}(t)\right)^{\prime}}{a_{2}(t) Z^{\prime}(t)}+\phi(t) \frac{\left(a_{1}(t)\left(a_{2}(t) Z^{\prime}(t)\right)^{\prime}\right)^{\prime}}{a_{2}(t) Z^{\prime}(t)} \\
& -\phi(t) \frac{a_{1}(t)\left(a_{1}(t)\left(a_{2}(t) Z^{\prime}(t)\right)^{\prime}\right)\left(a_{2}(t) Z^{\prime}(t)\right)^{\prime}}{\left(a_{2}(t) Z^{\prime}(t)\right)^{2}} .
\end{aligned}
$$

Since $\left(a_{1}(t)\left(a_{2}(t) Z^{\prime}(t)\right)^{\prime}\right)^{\prime}<0$, then $a_{1}(t)\left(a_{2}(t) Z^{\prime}(t)\right)^{\prime}$ is decreasing, so

$$
a_{2}(t) Z^{\prime}(t) \geq \int_{t_{1}}^{t} \frac{a_{1}(s)\left(a_{2}(s) Z^{\prime}(s)\right)^{\prime}}{a_{1}(s)} d s \geq a_{1}(t)\left(a_{2}(t) Z^{\prime}(t)\right)^{\prime} \int_{t_{1}}^{t} \frac{d s}{a_{1}(s)},
$$

which implies that

$$
\left(\frac{a_{2}(t) Z^{\prime}(t)}{\int_{t_{1}}^{t} d s / a_{1}(s)}\right)^{\prime} \leq 0
$$

Thus,

$$
Z(t)=Z\left(t_{2}\right)+\int_{t_{2}}^{t} \frac{a_{2}(s) Z^{\prime}(s)}{\int_{t_{1}}^{s} \frac{d u}{a_{1}(u)}} \frac{\int_{t_{1}}^{s} \frac{d u}{a_{1}(u)}}{a_{2}(s)} d s \geq \frac{a_{2}(t) Z^{\prime}(t)}{\int_{t_{1}}^{t} \frac{d u}{a_{1}(u)}} \int_{t_{2}}^{t} \frac{\int_{t_{1}}^{s} \frac{d u}{a_{1}(u)}}{a_{2}(s)} d s,
$$


for $t \geq t_{2} \geq t_{1}$.

It follows from $(E),(2.4)$, and (2.5) that

$$
\omega^{\prime}(t) \leq \frac{\phi^{\prime}(t)}{\phi(t)} \omega(t)-\frac{\omega^{2}(t)}{\phi(t) a_{1}(t)}-\phi(t) k q(t) p_{*}(t) \frac{Z(\sigma(t))}{a_{2}(t) Z^{\prime}(t)},
$$

that is,

$$
\omega^{\prime}(t) \leq \frac{\phi^{\prime}(t)}{\phi(t)} \omega(t)-\frac{\omega^{2}(t)}{\phi(t) a_{1}(t)}-\phi(t) k q(t) p_{*}(t) \frac{Z(\sigma(t))}{a_{2}(\sigma(t)) Z^{\prime}(\sigma(t))} \frac{a_{2}(\sigma(t)) Z^{\prime}(\sigma(t))}{a_{2}(t) Z^{\prime}(t)} .
$$

From (2.6) and (2.7) follows

$$
\begin{gathered}
\omega^{\prime}(t) \leq \frac{\phi^{\prime}(t)}{\phi(t)} \omega(t)-\frac{\omega^{2}(t)}{\phi(t) a_{1}(t)}-\phi(t) k q(t) p_{*}(t) \frac{\int_{t_{2}}^{\sigma(t)}\left(\frac{1}{a_{2}(s)} \int_{t_{1}}^{s} \frac{d u}{a_{1}(u)}\right) d s}{\int_{t_{1}}^{\sigma(t)} \frac{d u}{a_{1}(u)} \frac{\int_{t_{1}}^{\sigma(t)} \frac{d u}{a_{1}(u)}}{\int_{t_{1}}^{t} \frac{d u}{a_{1}(u)}}} \\
\left.=\frac{\phi^{\prime}(t)}{\phi(t)} \omega(t)-\frac{\omega^{2}(t)}{\phi(t) a_{1}(t)}-\phi(t) k q(t) p_{*}(t) \frac{1}{t_{2}(s)} \int_{t_{1}}^{s} \frac{d u}{a_{1}(u)}\right) d s \\
\int_{t_{1}}^{t} \frac{d u}{a_{1}(u)} \\
\leq-\left[\frac{\omega(t)}{\sqrt{\phi(t) a_{1}(t)}}-\frac{1}{2} \sqrt{\frac{a_{1}(t)}{\phi(t)}} \phi^{\prime}(t)\right]^{2}-\phi(t) q(t) k p_{*}(t) \Theta(t)+\frac{a_{1}(t)\left(\phi^{\prime}(t)\right)^{2}}{4 \phi(t)}
\end{gathered}
$$

which implies

$$
\omega^{\prime}(t) \leq-\phi(t) q(t) k p_{*}(t) \Theta(t)+\frac{a_{1}(t)\left(\phi^{\prime}(t)\right)^{2}}{4 \phi(t)}
$$

Integrating the last inequality from $t_{3}\left(>t_{2}\right)$ to $t$ we obtain

$$
\int_{t_{3}}^{t}\left(\phi(s) q(s) k p_{*}(s) \Theta(s)-\frac{a_{1}(s)\left(\phi^{\prime}(s)\right)^{2}}{4 \phi(s)}\right) d s \leq \omega\left(t_{3}\right) .
$$

Letting $t \rightarrow \infty$, it contradicts to $(2.2)$.

Assume the case (2) holds. Using the similar proof of [10, Lemma 2], we can get $\lim _{t \rightarrow \infty} x(t)=0$ due to condition (2.3).

Theorem 2. Let $0 \leq p(t) \leq p_{1} \leq 1$. If (1.2) holds and there exists a positive function $\varphi \in C^{1}\left(\left[t_{0}, \infty\right), \mathbb{R}\right)$, such that for all sufficiently large $t_{3}>t_{2}>t_{1} \geq t_{0}$, one has (2.2) and (2.3). If

$$
\limsup _{t \rightarrow \infty} \int_{t_{2}}^{t}\left(\delta(s) q(s) k p_{*}(s)\left(\int_{t_{1}}^{\sigma(s)} \frac{d v}{a_{2}(v)}\right)-\frac{1}{4 \delta(s) a_{1}(s)}\right) d s=\infty,
$$

where

$$
\delta(t):=\int_{t}^{\infty} \frac{1}{a_{1}(s)} d s
$$

holds for all constants $M>0$, then $(E)$ is almost oscillatory. 
P r o o f. Suppose that $x(t)$ is a positive solution of $(E)$. By condition (1.2), there exist three possible cases:

(1) $Z(t)>0, Z^{\prime}(t)>0,\left(a_{2}(t) Z^{\prime}(t)\right)^{\prime}>0,\left(a_{1}(t)\left(a_{2}(t) Z^{\prime}(t)\right)^{\prime}\right)^{\prime}<0$,

(2) $Z(t)>0, Z^{\prime}(t)<0,\left(a_{2}(t) Z^{\prime}(t)\right)^{\prime}>0,\left(a_{1}(t)\left(a_{2}(t) Z^{\prime}(t)\right)^{\prime}\right)^{\prime}<0$, and

(3) $Z(t)>0, Z^{\prime}(t)>0,\left(a_{2}(t) Z^{\prime}(t)\right)^{\prime}<0,\left(a_{1}(t)\left(a_{2}(t) Z^{\prime}(t)\right)^{\prime}\right)^{\prime}<0$, for $t \geq t_{1}, t_{1}$ is large enough.

In cases (1) and (2) we can obtain the conclusion of Theorem 2 by applying the proof of Theorem 1 . Assume that case (3) holds, $\left(a_{1}(t)\left(a_{2}(t) Z^{\prime}(t)\right)^{\prime}\right)^{\prime}<0$ and $a_{1}(t)\left(a_{2}(t) Z^{\prime}(t)\right)^{\prime}$ is nonincreasing. Thus, we get

$$
a_{1}(s)\left(a_{2}(s) Z^{\prime}(s)\right)^{\prime} \leq a_{1}(t)\left(a_{2}(t) Z^{\prime}(t)\right)^{\prime}, \quad s \geq t \geq t_{1} .
$$

Dividing the above inequality by $a_{1}(s)$ and integrating from $t$ to $l$, we obtain

$$
a_{2}(l) Z^{\prime}(l) \leq a_{2}(t) Z^{\prime}(t)+a_{1}(t)\left(a_{2}(t) Z^{\prime}(t)\right)^{\prime} \int_{t}^{l} \frac{d s}{a_{1}(s)} .
$$

Letting $l \rightarrow \infty$, we have

$$
0 \leq a_{2}(t) Z^{\prime}(t)+a_{1}(t)\left(a_{2}(t) Z^{\prime}(t)\right)^{\prime} \int_{t}^{\infty} \frac{d s}{a_{1}(s)}
$$

that is,

$$
-\frac{a_{1}(t)\left(a_{2}(t) Z^{\prime}(t)\right)^{\prime}}{a_{2}(t) Z^{\prime}(t)} \int_{t}^{\infty} \frac{d s}{a_{1}(s)} \leq 1
$$

Now define $\varphi$ as

$$
\varphi(t):=\frac{a_{1}(t)\left(a_{2}(t) Z^{\prime}(t)\right)^{\prime}}{a_{2}(t) Z^{\prime}(t)}, \quad t \geq t_{1} .
$$

Then $\varphi(t)<0$ for $t \geq t_{1}$. Therefore, by (2.9) and (2.10), we obtain

$$
-\delta(t) \varphi(t) \leq 1
$$

Differentiating (2.10) gives

$$
\varphi^{\prime}(t)=\frac{\left(a_{1}(t)\left(a_{2}(t) Z^{\prime}(t)\right)^{\prime}\right)^{\prime}}{a_{2}(t) Z^{\prime}(t)}-\frac{a_{1}(t) a_{1}(t)\left(a_{2}(t) Z^{\prime}(t)\right)^{\prime}\left(a_{2}(t) Z^{\prime}(t)\right)^{\prime}}{\left(a_{2}(t) Z^{\prime}(t)\right)^{2}} .
$$

Now $Z^{\prime}(t)>0$, so from $(E)$ and $(2.4)$ we have

$$
\varphi^{\prime}(t) \leq-q(t) k p_{*}(t) \frac{Z(\sigma(t))}{a_{2}(t) Z^{\prime}(t)}-\frac{a_{1}(t) a_{1}(t)\left(a_{2}(t) Z^{\prime}\right)^{\prime}\left(a_{2}(t) Z^{\prime}(t)\right)^{\prime}}{\left(a_{2}(t) Z^{\prime}(t)\right)^{2}} .
$$

In view of case (3), we see that

$$
Z(t) \geq a_{2}(t) \int_{t_{1}}^{t} \frac{d s}{a_{2}(s)} Z^{\prime}(t)
$$


Thus, $\left(\frac{Z(t)}{\int_{t_{1}}^{t} d s / a_{2}(s)}\right)^{\prime} \leq 0$ implies

$$
\frac{Z(\sigma(t))}{Z(t)} \geq \frac{\int_{t_{1}}^{\sigma(t)} \frac{d s}{a_{2}(s)}}{\int_{t_{1}}^{t} \frac{d s}{a_{2}(s)}} .
$$

From (2.10) and (2.12)-(2.14) we have

$$
\varphi^{\prime}(t) \leq-q(t) k p_{*}(t) \int_{t_{1}}^{\sigma(t)} \frac{d s}{a_{2}(s)}-\frac{\varphi^{2}(t)}{a_{1}(t)} .
$$

Multiplying the above inequality by $\delta(t)$ and integrating it from $t_{2}\left(>t_{1}\right)$ to $t$, we have

$$
\begin{gathered}
\varphi(t) \delta(t)-\varphi\left(t_{2}\right) \delta\left(t_{2}\right)+\int_{t_{2}}^{t} \delta(s) k q(s) p_{*}(s)\left(\int_{t_{1}}^{\sigma(s)} \frac{d v}{a_{2}(v)}\right) d s \\
+\int_{t_{2}}^{t} \frac{\varphi^{2}(s) \delta(s)}{a_{1}(s)} d s+\int_{t_{2}}^{t} \frac{\varphi(s)}{a_{1}(s)} d s \leq 0 \\
\varphi(t) \delta(t)-\varphi\left(t_{2}\right) \delta\left(t_{2}\right)+\int_{t_{2}}^{t} \delta(s) k q(s) p_{*}(s)\left(\int_{t_{1}}^{\sigma(s)} \frac{d v}{a_{2}(v)}\right) d s-\int_{t_{2}}^{t} \frac{d s}{4 \delta(s) a_{1}(s)} \\
+\int_{t_{2}}^{t}\left[\sqrt{\frac{\delta(s)}{a_{1}(s)}} \varphi(s)+\frac{1}{2} \frac{1}{\sqrt{a_{1}(s) \delta(s)}}\right]^{2} d s \leq 0,
\end{gathered}
$$

from which it follows that

$$
\int_{t_{2}}^{t}\left(\delta(s) q(s) k p_{*}(s)\left(\int_{t_{1}}^{\sigma(s)} \frac{d v}{a_{2}(v)}\right)-\frac{1}{4 \delta(s) a_{1}(s)}\right) d s \leq 1+\varphi\left(t_{2}\right) \delta\left(t_{2}\right)
$$

due to (2.11). Letting $t \rightarrow \infty$, we come to the contradiction (2.8). Then the result of the Theorem follows.

\section{Examples}

In this section we will present some examples to illustrate the main results.

Example 1. Consider a third-order neutral differential equation

$$
\left(t^{-1 / 2}\left(t^{1 / 2}\left[x(t)+\frac{1}{4} x^{3 / 5}(t-1)\right]^{\prime}\right)^{\prime}\right)^{\prime}+\frac{\lambda}{t^{1 / 2}} x(t-2)=0, \quad t \geq 1,
$$

where $\lambda>0$ is a constant. Let $\alpha=3 / 5, a_{1}(t)=t^{-1 / 2}, a_{2}(t)=t^{1 / 2}, p(t)=1 / 4, q(t)=\frac{\lambda}{t^{1 / 2}}$, $\tau(t)=t-1$, and $\sigma(t)=t-2$. We obtain $p_{*}(t)=1-\frac{1 / 4}{M^{2 / 5}}$,

$$
\int_{t_{0}}^{\infty} \frac{1}{a_{2}(v)} \int_{v}^{\infty} \frac{1}{a_{1}(u)}\left[\int_{u}^{\infty} \frac{\lambda}{s^{1 / 2}} d s\right] d u d v=\infty
$$


and

$$
\Theta(t)=\frac{\int_{t_{2}}^{\sigma(t)}\left(\frac{1}{a_{2}(s)} \int_{t_{1}}^{s} \frac{d u}{a_{1}(u)}\right) d s}{\int_{t_{1}}^{t} \frac{d u}{a_{1}(u)}}=\frac{(t-2)^{3}+6(t-2)^{1 / 2} t_{1}^{1 / 2}-3 c}{t^{3 / 2}-t_{1}^{3 / 2}},
$$

where $c=t_{2}^{3} / 3+2 t_{1}^{3 / 2} t_{2}^{1 / 2}$. Pick $\phi(t)=1$, then

$$
\int_{t_{3}}^{\infty} q(s) p_{*}(s) \Theta(s) d s=\frac{\lambda}{3}\left(1-\frac{1 / 4}{M^{2 / 5}}\right) \int_{t_{3}}^{\infty} \frac{(s-2)^{3}+6(s-2)^{1 / 2} t_{1}^{1 / 2}-3 c}{s^{2}-s^{1 / 2} t_{1}^{3 / 2}}=\infty,
$$

if $1 / 4<M^{2 / 5}$. Hence, by Theorem 1, every solution of equation (3.1) is either oscillatory or converges to zero as $t \rightarrow \infty$ when $1 / 4<M^{2 / 5}$.

Example 2. Consider a third-order neutral differential equation

$$
\left(t^{2}\left[x(t)+\frac{1}{2} x^{1 / 3}(t / 8)\right]^{\prime \prime}\right)^{\prime}+\frac{1}{t}\left(1+\frac{2}{27} t^{2 / 3}\right) x(t / 2)=0, \quad t \geq 1
$$

Let $\alpha=1 / 3, a_{1}(t)=t^{2}, a_{2}(t)=1, p(t)=1 / 2, q(t)=1 / t\left(1+2 / 27 t^{2 / 3}\right), \tau(t)=t / 8$ and $\sigma(t)=t / 2$. We obtain

$$
p_{*}(t)=1-\frac{1 / 2}{M^{2 / 3}}, \quad \delta(t)=\int_{t}^{\infty} \frac{d s}{s^{2}}=\frac{1}{t}
$$

and

$$
\int_{t_{1}}^{t / 2} \frac{d s}{a_{2}(s)}=\frac{1}{2}\left(t-2 t_{1}\right)
$$

then

$$
\begin{gathered}
\int_{t_{2}}^{\infty}\left(\delta(s) q(s) p_{*}(s)\left(\int_{t_{1}}^{\sigma(s)} \frac{d v}{a_{2}(v)}\right)-\frac{1}{4 \delta(s) a_{1}(s)}\right) d s \\
=\int_{t_{2}}^{\infty}\left(\left(1-0.5 M^{-2 / 3}\right)\left[\frac{1}{2 s}+\frac{1}{27} s^{-1 / 3}-\frac{t_{1}}{s^{2}}-\frac{2 t_{1}}{27} s^{-4 / 3}\right]-\frac{1}{4 s}\right) d s=\infty,
\end{gathered}
$$

if $0.5<M^{2 / 3}$. Hence, by Theorem 2, every solution of equation (3.2) is either oscillatory or converges to zero as $t \rightarrow \infty$ when $0.5<M^{2 / 3}$ and $x(t)=t^{-1}$ is such a solution of (3.2).

\section{Acknowledgement}

The authors would like to thank very much the anonymous referees for valuable suggestions, corrections and comments, which result in a great improvement of the original manuscript.

\section{References}

1. Agarwal R.P., Bohner M., Li T., Zhang C. Oscillation of second-order Emden-Fowler neutral delay differential equations // Ann. Math. Pura Appl., 2004. Vol. 193, no. 6. P. 18611875. DOI: $10.1007 / \mathrm{s} 10231-013-0361-7$

2. Agarwal R.P., Bohner M., Li T., Zhang C. A new approach in the study of oscillatory behavior of even-order neutral delay differential equations // Appl. Math. Comput., 2013. Vol. 225. P. 787-794. DOI: 10.1016/j.amc.2013.09.037 
3. Dix J.G. Oscillation of solutions to a neutral differential equation involving an $n$-order operator with variable coefficients and a forcing term // Differ. Equ. Dyn. Syst., 2014. Vol. 22, no. 1. P. 15-31. DOI: $10.1007 / \mathrm{s} 12591-013-0160-\mathrm{z}$

4. Ganesan V. and M. Sathish Kumar Oscillation criteria for second-order neutral differential equations // Int. J. of Pure and Appl. Math., 2017. Vol. 113, no. 12. P. 151-159.

5. Ganesan V. and M. Sathish Kumar Oscillation of certain third order nonlinear differential equation with neutral terms // Bangmod Int. J. of Math. Comp. Sci., 2017. Vol. 3, no. 1-2. P. 53-60.

6. Ganesan V. and M. Sathish Kumar Oscillation theorems for third-order retarded differential equations with a sublinear neutral term // Int. J. of Pure and Appl. Math., 2017. Vol. 114, no. 5, P. 63-70.

7. Graef J.R., Savithri R. and Thandapani E. Oscillatory properties of third order neutral delay differential equations // Discrete and Continuous Dynamical Systems A, 2003. P. 342350 .

8. Thandapani E. and Li T. On the oscillation of third-order quasi-linear neutral functional differential equations // Archivum Mathematicum, 2011. Vol. 47. P. 181-199.

9. Tamilvanan S., Thandapani E., Džurina J. Oscillation of second order nonlinear differential equation with sublinear neutral term // Differential Equations and Applications, 2016. Vol. 9, no. 1. P. 29-35. DOI: dx.doi.org/10.7153/dea-09-03

10. Baculíková B. and Džurina J. Oscillation of third-order neutral differential equations // Math. Comput. Modelling, 2010. Vol. 52. P. 215-226. DOI: 10.1016/j.mcm.2010.02.011

11. Candan T., Dahiya R.S. Oscillation of third order functional differential equations with delay // Electron. J. Diff. Eqns. Conference, 2003. Vol. 10. P. 79-88.

12. Candan T., Dahiya R.S. Functional differential equations of third order // Electron. J. Diff. Eqns. Conference, 2005. Vol. 12. P. 47-56.

13. Lin X. and Tang $\mathbf{X}$. Oscillation of solutions of neutral differential equations with a superlinear neutral term // Applied Mathematics Letters, 2007. Vol. 20. P. 1016-1022. DOI: $10.1016 / \mathrm{j} . \mathrm{aml} .2006 .11 .006$

14. Agarwal R.P., Bohner M., Li T. and Zhang C. Oscillation of second order differential equations with a sublinear neutral term // Carpathian J. Math, 2014. Vol. 30. P. 1-6. 